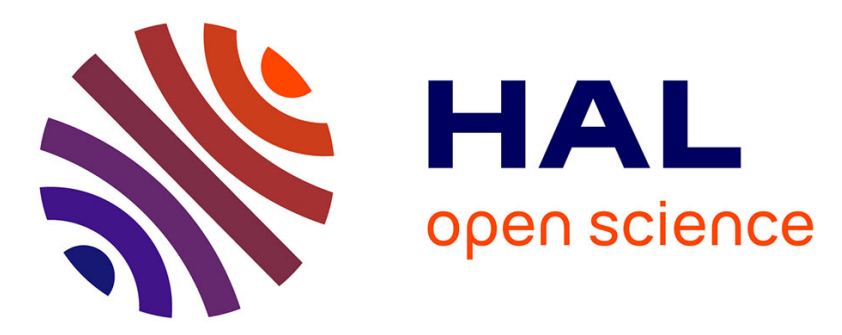

\title{
Régimes convectifs instationnaires dans l'air en cavité à grands facteurs d'aspect : résultats expérimentaux
}

B. Martinet, P. Haldenwang, G. Labrosse, J.C. Payan, R. Payan

\section{To cite this version:}

B. Martinet, P. Haldenwang, G. Labrosse, J.C. Payan, R. Payan. Régimes convectifs instationnaires dans l'air en cavité à grands facteurs d'aspect : résultats expérimentaux. Journal de Physique Lettres, 1982, 43 (6), pp.161-169. 10.1051/jphyslet:01982004306016100 . jpa-00232026

\section{HAL Id: jpa-00232026 https://hal.science/jpa-00232026}

Submitted on 1 Jan 1982

HAL is a multi-disciplinary open access archive for the deposit and dissemination of scientific research documents, whether they are published or not. The documents may come from teaching and research institutions in France or abroad, or from public or private research centers.
L'archive ouverte pluridisciplinaire HAL, est destinée au dépôt et à la diffusion de documents scientifiques de niveau recherche, publiés ou non, émanant des établissements d'enseignement et de recherche français ou étrangers, des laboratoires publics ou privés. 


\title{
LE JOURNAL DE PHYSIQUE-LETTRES
}

J. Physique - LETTRES 43 (1982) L-161 - L-169

15 MARS 1982, PAGE L-161

Classification

Physics Abstracts

$47.25 \mathrm{Q}$

\section{Régimes convectifs instationnaires dans l'air en cavité à grands facteurs d'aspect : résultats expérimentaux}

\author{
B. Martinet, P. Haldenwang, G. Labrosse, J. C. Payan et R. Payan \\ Département d'Héliophysique $\left(^{*}\right)$, \\ Université de Provence, 13397 Marseille Cedex 13, France
}

(Reçu le 18 décembre 1981, révisé le 19 janvier 1982, accepté le 25 janvier 1982)

\begin{abstract}
Résumé. - Nous présentons des résultats expérimentaux sur la transition vers la turbulence d'écoulement convectif de l'air en cavité parallélépipédique horizontale de facteur d'aspect 14 . On observe des régimes dépendant du temps du type monopériodique et quasipériodique. La fréquence de l'oscillateur monopériodique dépend linéairement du nombre de Rayleigh pour la gamme étudiée : $14000<R a<30000$.

Abstract. - Experimental results are presented for the transition to turbulence of the air convective flow in horizontal parallelepipedic box of aspect ratio 14 . We observe periodic and quasiperiodic time dependence. The monoperiodic oscillator frequency depends linearly of the Rayleigh number within the range 14000,30000 .
\end{abstract}

1. Introduction. - L'analyse de la transition vers la turbulence de l'instabilité de RayleighBenard suscite depuis quelques années un intérêt théorique et expérimental croissant [1-7].

Il a été souligné que cette transition peut s'effectuer par de multiples voies dont le choix dépend fortement de l'histoire du système et de paramètres comme le nombre de Prandtl et le facteur d'aspect.

Notre préoccupation solaire (modélisation de convertisseurs héliothermiques plans) nous a conduit à nous intéresser à cette transition dans le cas de l'air (Prandtl $=0,71)$ confiné en cavités horizontales à facteur d'aspect (longueur/hauteur) nettement supérieur à 1 .

Les résultats présentés à ce jour concernant l'instabilité de Rayleigh-Benard s'intéressent soit à des cavités cylindriques pour une grande gamme de facteurs d'aspect [2,4], soit à des géométries parallélépipédiques de facteur d'aspect proche de l'unité [1-2], ou grand [7].

Notre étude expérimentale vient s'insérer dans cet ensemble. La cavité parallélépipédique

(*) ERA CNRS no 538 . 
choisie, de facteur d'aspect 14 présente un rapport longueur sur largeur de 3,3 favorisant un écoulement à caractère bidimensionnel.

Nous verrons que ces résultats, en accord avec ceux de Willis et al. [7], contredisent l'hypothèse selon laquelle un facteur d'aspect important suffit pour qu'il y ait transition directe du régime conductif vers un régime turbulent [4].

2. Dispositif expérimental. - 2.1 CAVITÉ. - L'expérience a été réalisée à partir d'une cavité parallélépipédique de dimension horizontale $28 \times 8,5 \mathrm{~cm}^{2}$ et de hauteur $2 \mathrm{~cm}$ constituée par deux plaques horizontales en cuivre massif et par des parois latérales verticales en verre de très bonne qualité optique pour les grands côtés de la cavité (direction $\mathrm{O} x$ ) et en résine epoxy pour les petits côtés (direction $\mathrm{O} y$ ) figure 1. Nous définissons un facteur d'aspect horizontal

$$
\Gamma_{\mathrm{h}}=\frac{\text { longueur }}{\text { largeur }}=3,3 \text { et } \text { vertical } \Gamma_{\mathrm{v}}=\frac{\text { longueur }}{\text { hauteur }}=14 .
$$

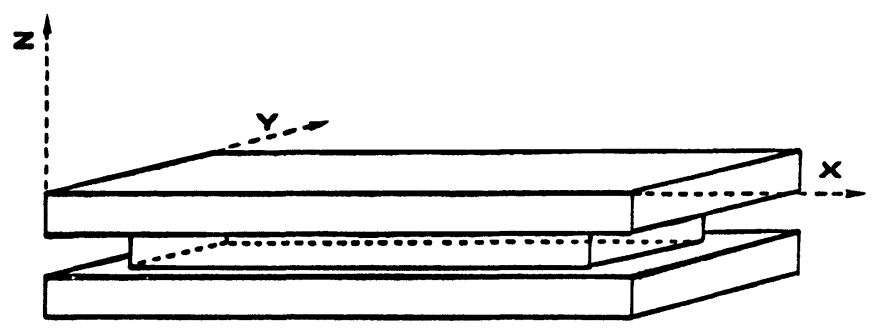

Fig. 1. - Schémas de la cavité expérimentale.

[Block diagram of the experimental box.]

Le choix de ces matériaux est lié, d'une part à la méthode de visualisation présentée ci-après, et d'autre part aux conditions de flux transverse les plus faibles possibles sur les deux autres parois.

Les deux plaques horizontales sont maintenues isothermes, l'une, la plaque supérieure, étant refroidie par circulation d'eau, et l'autre étant chauffée par effet Joule et régulée en température. L'isothermie des plaques inférieure et supérieure contrôlée par Thermographie Infrarouge ainsi que la stabilité en température sont assurées à $0,1^{\circ} \mathrm{C}$.

Les différences de température $(\Delta T)$ entre les deux plaques se situent pour l'étude effectuée entre 19 et $56^{\circ} \mathrm{C}$. Dans ces conditions expérimentales, la variation du nombre de Prandtl du fluide (air), confiné dans la cavité, n'excède pas $1 \%$.

La prise en compte de toutes les erreurs (dimensions de la cavité, température et constante physique du fluide) conduit à une incertitude relative sur la mesure du nombre de Rayleigh de $2 \%$.

2.2 Méthode de visualisation. - L'analyse de l'écoulement est réalisée à l'aide d'un montage d'interférométrie différentielle (Fig. 2) donnant la vision complète en temps réel d'un champ d'isogradient intégré le long de la direction parallèle au petit côté $\mathrm{O} y$ de la cavité.

Le principe du montage est le suivant :

La lumière issue d'un arc ponctuel à vapeur de mercure $(\mathrm{S})$ polarisée rectilignement en $\mathrm{p}$ est condensée sur un prisme de Wollaston $\mathrm{W}_{1}$, dont les axes optiques sont à $45^{\circ}$ de la direction de polarisation, qui divise chaque rayon en deux rayons polarisés perpendiculairement. Ces deux rayons traversent à l'aller et au retour la veine de mesure à visualiser placée contre un miroir sphérique concave $\mathrm{m}_{1}$, de grande focale $(3 \mathrm{~m})$. Les deux rayons sont ensuite confondus après condensation sur un second prisme de Wollaston $\mathrm{W}_{2}$ identique à $\mathrm{W}_{1}$, et de même orientation.

Un analyseur a croisé avec le polariseur p permet aux deux rayons d'interférer. 


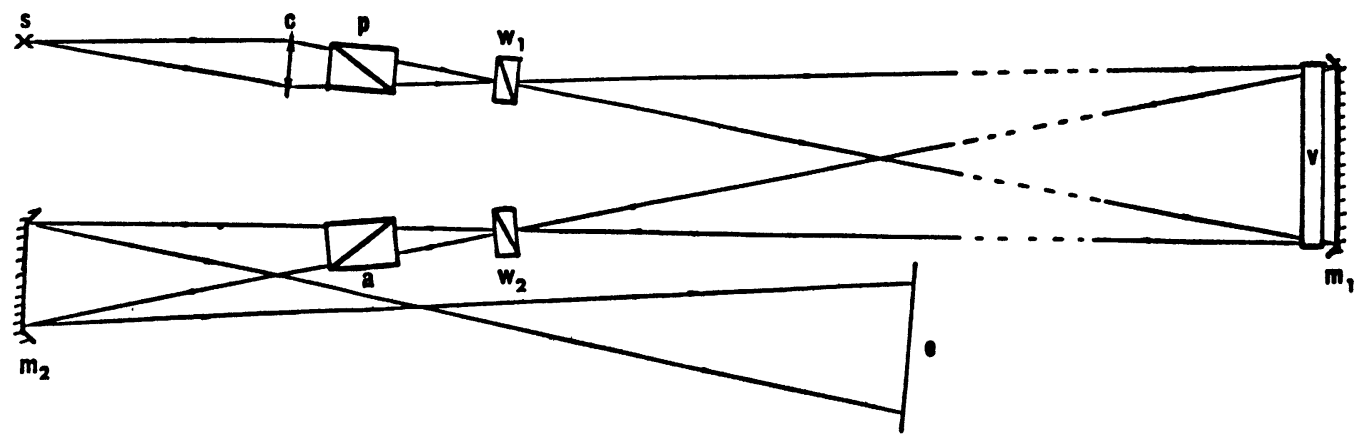

INTERFEROMETRE DIFFERENTIEL

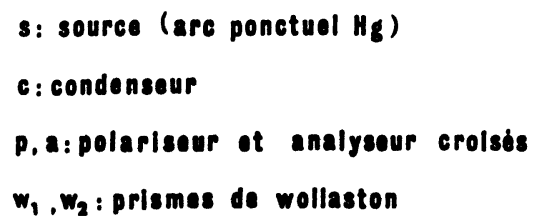

$V$ : section d'observation

$m_{1}, m_{2}:$ mirolrs concaves

o: deran de observation

Fig. 2. - Schémas du montage optique.

[Optical set up.]

Le miroir concave $\mathrm{m}_{2}$ conjugue le plan d'observation e avec la veine de mesure V.

L'interférogramme obtenu sur e nous fournit la cartographie en temps réel du champ des isogradients de température dans la direction de séparation des rayons.

Une simple rotation simultanée de $\mathrm{p}, \mathrm{W}_{1}, \mathrm{~W}_{2}$ et a autour de l'axe optique du système permet de modifier la direction de séparation et donc de déterminer les isogradients de l'écoulement dans les différentes directions du plan perpendiculaire à l'axe optique.

La sensibilité du dispositif dans le cas d'une veine de mesure de $0,1 \mathrm{~m}$, le long de l'axe optique du montage, est telle que l'on change l'ordre d'interférence d'une unité pour une variation du gradient de température de $1000^{\circ} \mathrm{C} . \mathrm{m}^{-1}$.

L'utilisation d'une source polychromatique permet d'augmenter notablement cette sensibilité. En figure 3, nous présentons les clichés d'une partie du champ de l'écoulement en isogradients horizontaux (a) et verticaux (b), pour un nombre de Rayleigh de 20000.

La mesure de la dépendance temporelle de ces écoulements est obtenue à l'aide d'un photomultiplicateur visant à travers une petite ouverture la zone centrale de l'un des rouleaux (voir repère sur le cliché 3 b). La surface de l'ouverture du photomultiplicateur est de $0,8 \mathrm{~mm}^{2}$ et la surface d'un rouleau dans l'espace image est de $20 \mathrm{~mm}^{2}$.

Le signal analogique en sortie du photomultiplicateur est amplifié, enregistré et traité numériquement. Le temps de prise de données est de $10 \mathrm{~min}$. avec 4096 points échantillons.

3. Présentation des résultats. - Dans une cavité de facteurs d'aspect $\Gamma_{\mathrm{v}}=10, \Gamma_{\mathrm{h}}=2,5$ comparables aux nôtres, et pour de l'azote, Oertel [6] a observé l'apparition d'un régime dépendant du temps au-delà de $R a / R a_{\mathrm{c}}=6,6$. Dans nos expériences, nous observons un régime dépendant du temps à partir de $R a / R a_{\mathrm{c}}=7,6$. En tenant compte des observations expérimentales de Willis et al. [7] (dépendance rapide de l'amplitude temporelle avec le nombre de Rayleigh) et du bruit de fond non négligeable de la méthode interférométrique dans le cas de l'air (Fig. 4), cette valeur ne peut que correspondre à une surestimation du seuil. Cependant, l'écart aux mesures de ce seuil $\left(R a / R a_{\mathrm{c}} \cong 3\right)$ présenté dans d'autres expériences [2,7], pourrait être imputable aux facteurs 


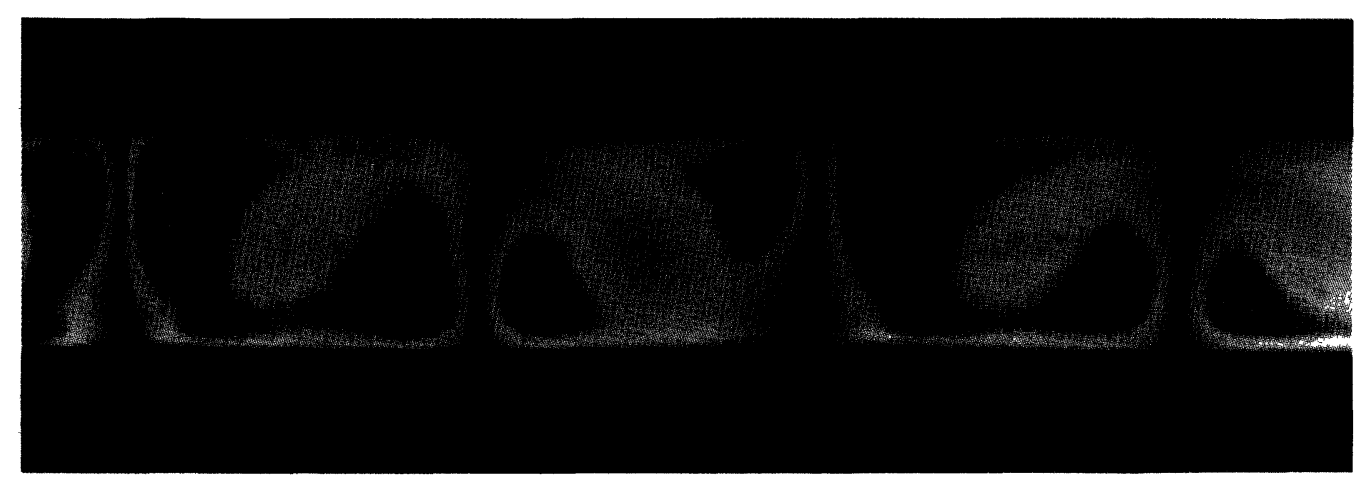

(a)

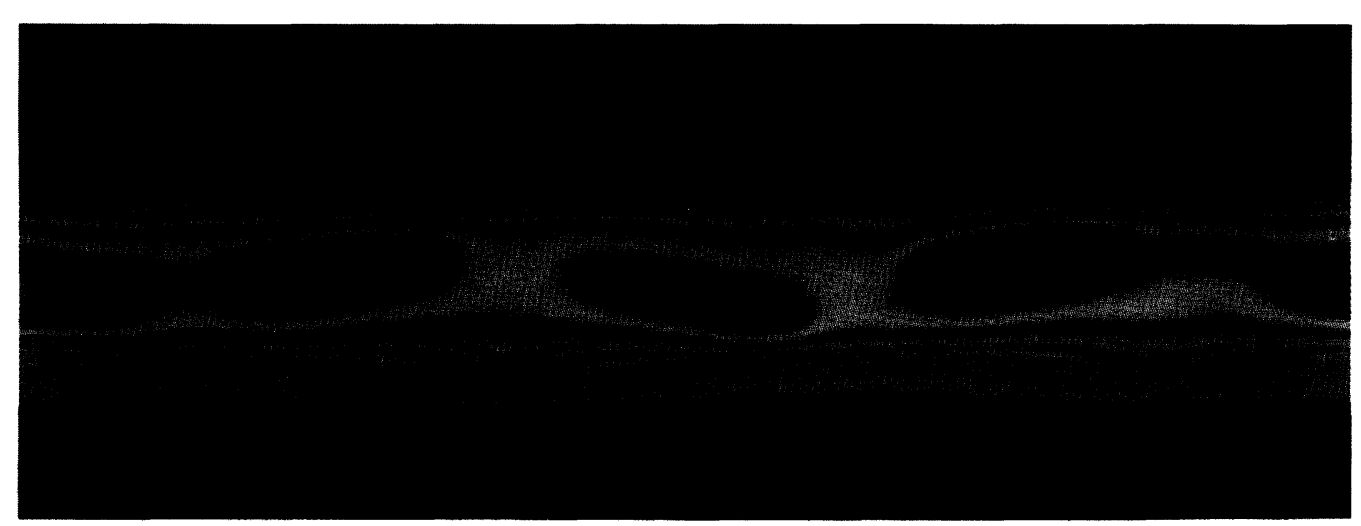

(b)

Fig. 3. - Image interférométrique des lignes d'isogradients de température : (a) horizontal, (b) vertical. [Interferometric images of the temperature isogradient lines : (a) horizontal, (b) vertical.] 


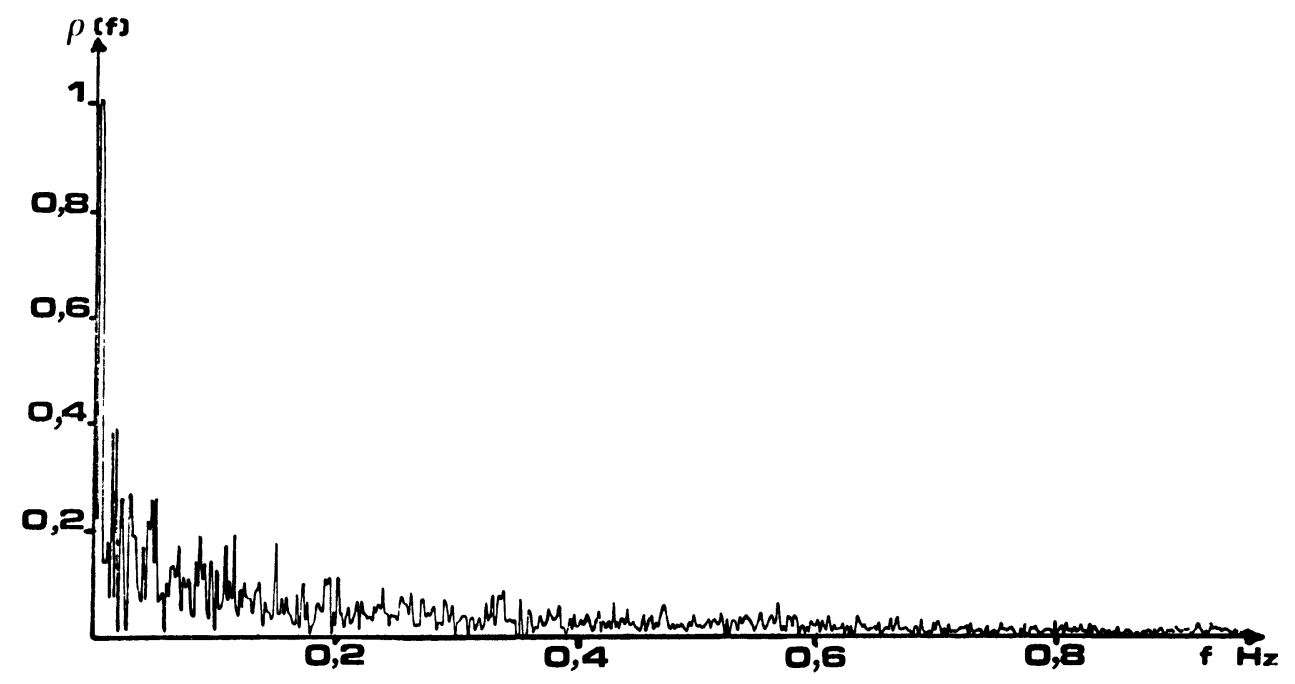

Fig. 4. - Spectre d'amplitude du bruit de fond de la chaîne de mesure.

[Amplitude spectrum of the experimental set up.]

d'aspect horizontaux $\Gamma_{\mathrm{h}}$ différents des nôtres. Comme dans d'autres expériences relatives aux régimes dépendant du temps [1-2], nous avons aussi constaté que l'historique du système intervient dans le choix du régime établi. Délibérément, nous nous proposons, pour l'instant, de ne pas prendre en compte cet aspect du problème mais seulement de répertorier les différents régimes observés.

Nous rapportons ici les résultats relatifs à une cinquantaine de mesures réparties sur un intervalle de nombre de Rayleigh compris entre 14000 et 30 000. Dans cette gamme de nombre de Rayleigh, les interférogrammes obtenus ont montré l'existence d'une structure à 10 rouleaux dont la distribution en température a un comportement temporel pulsant. La position du centre de ces rouleaux reste fixe.

Au-delà d'un nombre de Rayleigh de 20 000, la disposition géométrique régulière de ces 10 rouleaux, observée sur les interférogrammes, est significativement affectée. Nous interprétons cela comme l'apparition d'une tridimensionnalité dans l'écoulement.

Comme dans d'autres expériences relatives aux régimes dépendant du temps [1,2], nous avons aussi constaté que l'historique du système intervient dans le choix du régime établi. De l'ensemble des états observés se dégagent les catégories de spectres suivantes :

1. SPECTRES MONO OU QUASIPÉRIODIQUeS. - Ces spectres sont analogues à ceux que l'on rencontre dans la littérature $[1,2]$. Ils présentent les aspects suivants (Fig. 5) :

a) Aspect monopériodique : exemple, spectre I où la fréquence fondamentale est de $349 \mathrm{mHz}$.

b) Aspect quasipériodique : exemple, spectre II avec $f_{1}=426 \mathrm{mHz}$ (choisie par son amplitude dominante et son satellite $2 f_{1}$ ) et $f_{2}=168 \mathrm{mHz}$, raie de plus basse fréquence ; on note sur ce spectre un couplage simple du type $n f_{1}-f_{2}(n=1,2)$. D'autres satellites et raies couplés $\left(3 f_{1}\right.$, $\left.4 f_{1}, \ldots, 3 f_{1}-f_{2}, \ldots\right)$ sont présents sur nos spectres mais non représentés ici.

Pour l'ensemble de ces spectres, les raies se présentent couplées avec une bande basse fréquence, ce couplage très faible dans le cas des spectres I et II peut se trouver tout-à-fait remarquable dans d'autres cas.

On a même observé des spectres monopériodiques couplés avec une raie très bien résolue basse fréquence : exemple, spectre III avec $f_{1}=375 \mathrm{mHz}$ et $f_{3}=18 \mathrm{mHz}$. 

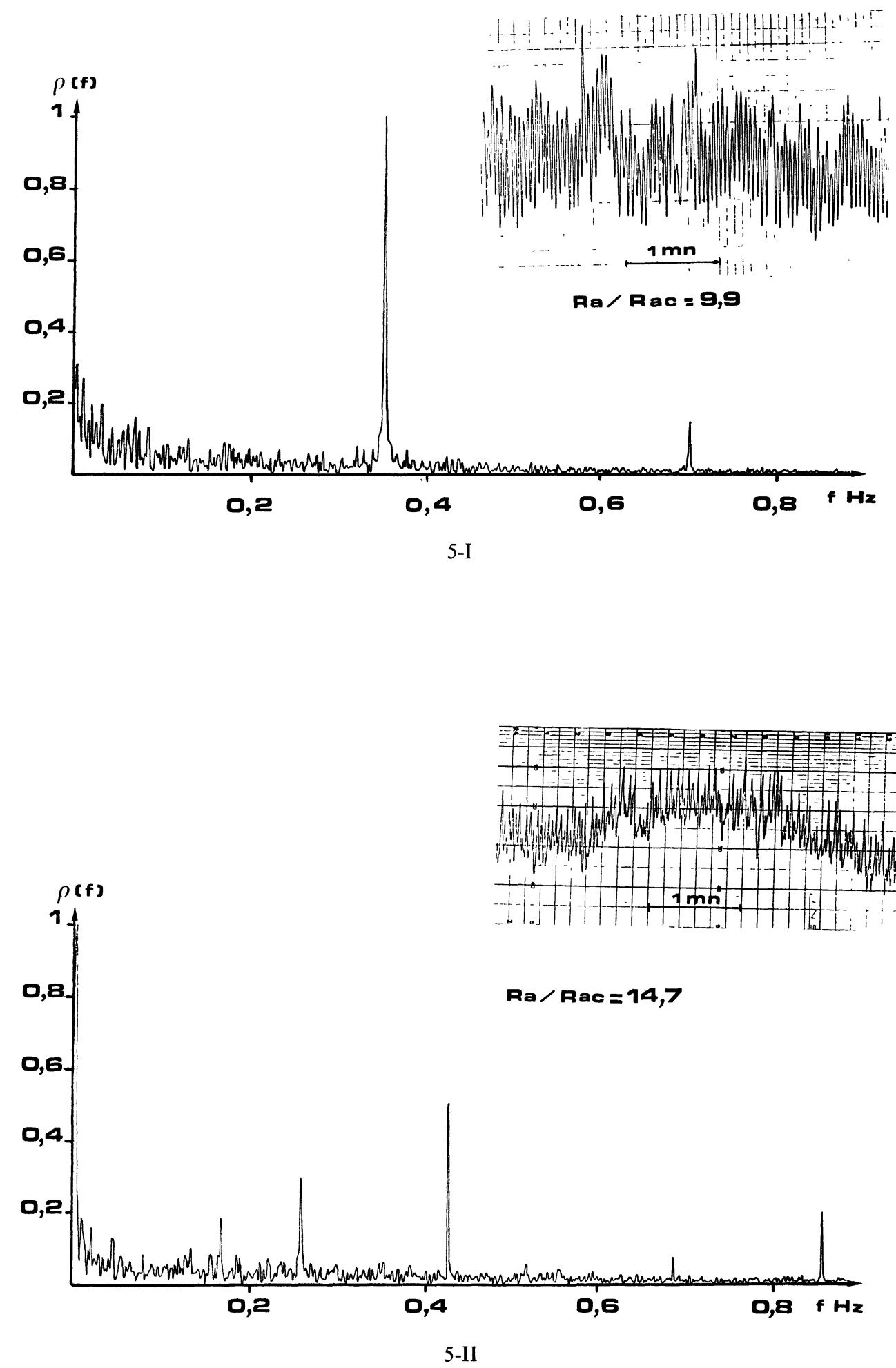

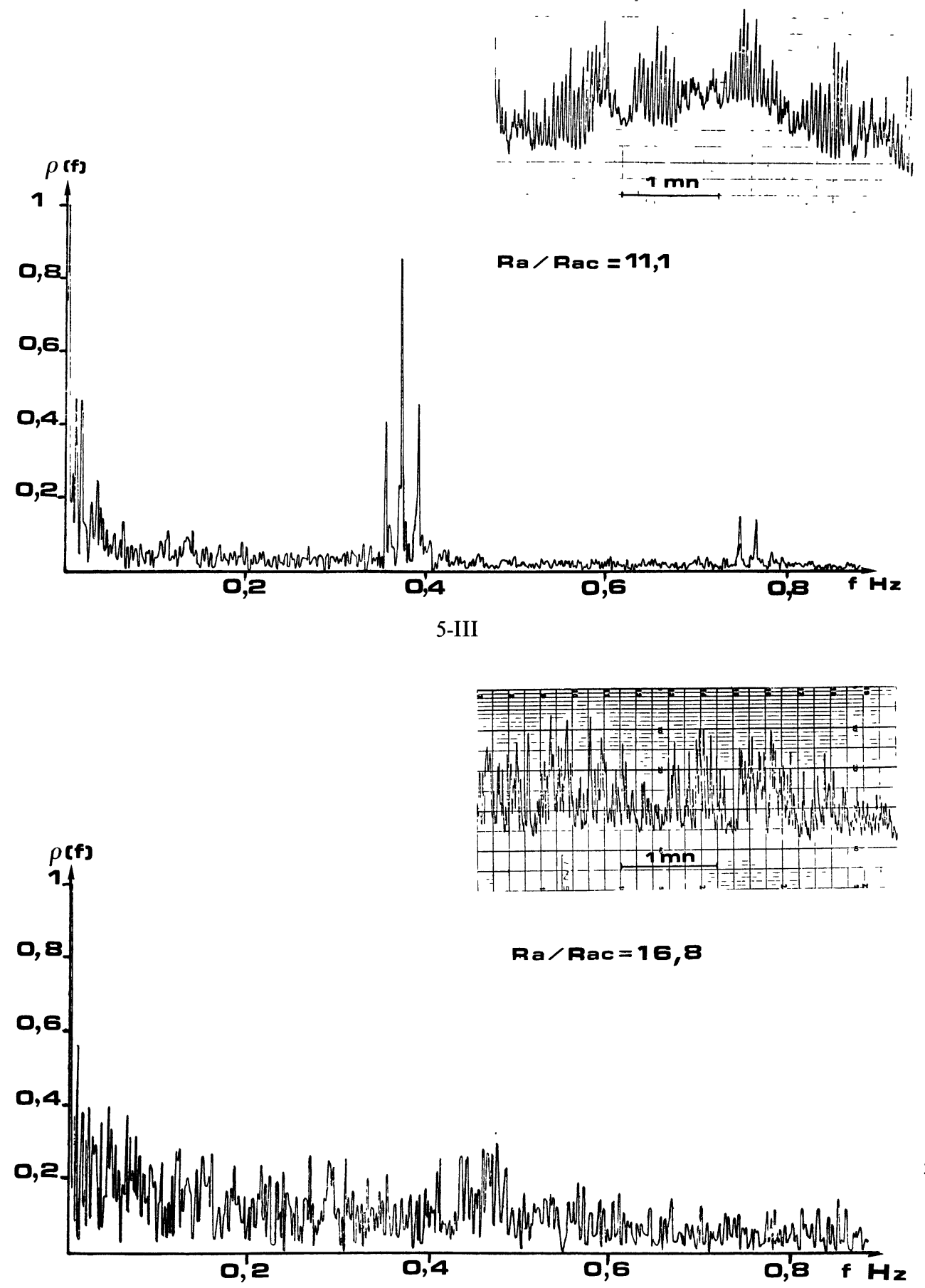

Fig. 5. - Spectre d'amplitude et dépendance temporelle de la température correspondante : (I) Spectre monopériodique; (II) Spectre quasipériodique; (III) Spectre monopériodique couplé avec une raie basse fréquence; (IV) Spectre chaotique.

[Amplitude spectrum and the corresponding temperature time dependence : (I) Monoperiodic spectrum; (II) Quasiperiodic spectrum ; (III) Coupled monoperiodic low frequency ray spectrum; (IV) Chaotic spectrum.] 
2. SpeCtres ChaOtiQues : exemple, spectre IV. - Ce spectre présente d'une manière à peine discernable l'emplacement de la fréquence $f_{1}$ (environ $470 \mathrm{mHz}$ ).

Malgré la grande diversité des états observables pour un nombre de Rayleigh donné, on constate que ce dernier reste le paramètre unificateur pour les fréquences observées.

Sur le graphe de la figure 6, nous présentons pour tous les régimes observés la dépendance en fonction du nombre de Rayleigh de la fréquence $f_{1}$ et de son premier harmonique, ainsi que la fréquence $f_{2}$. On notera sur ce graphe la permanence de l'oscillateur $f_{1}$, dont la fréquence suit avec une bonne approximation une loi linéaire de variation en nombre de Rayleigh.

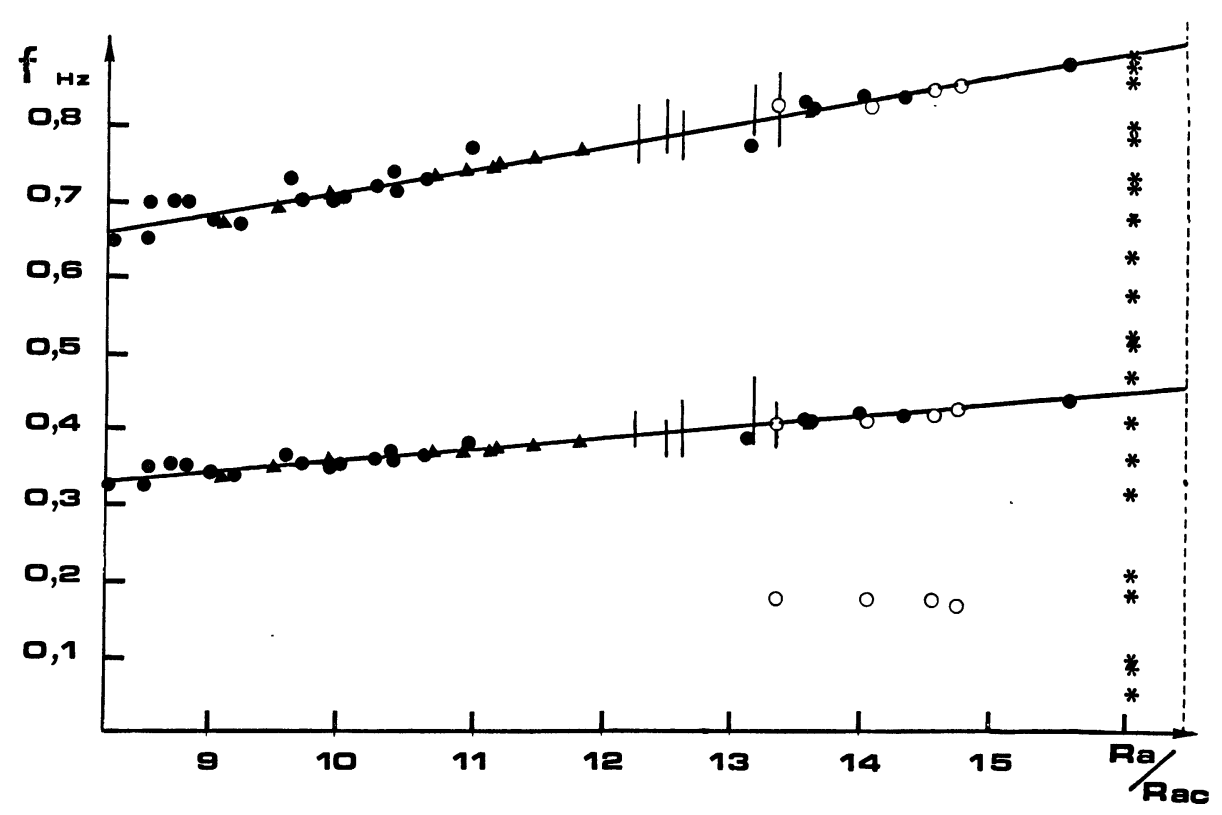

Fig. 6. - Variation de $f_{1}, 2 f_{1}, f_{2}$ avec le nombre de Rayleigh pour les spectres suivants : - monopériodique (Spectre I); O quasipériodique (Spectre II); $\Delta$ monopériodique couplé bande B.F.; monopériodique couplé raie B.F. (Spectre III); * Seul cas de spectre observé présentant une multiplicité de raies (deux fréquences fondamentales différentes de $f_{1}$ et $f_{2}$ et leurs harmoniques). La ligne en pointillés pour $R a / R a_{\mathrm{c}}=16,8$ donne la limite inférieure d'observation des spectres chaotiques (Spectre IV).

$\left[f_{1}, 2 f_{1}, f_{2}\right.$ variation versus the Rayleigh number for the following spectra : monoperiodic (Spectrum I) ; O quasiperiodic (Spectrum II); $\boldsymbol{\Delta}$ coupled monoperiodic low frequency band; | coupled monoperiodic low frequency ray (Spectrum III); * The only observed case of multiline spectrum (two fundamental frequencies different from $f_{1}$ and $f_{2}$ and their harmonics). The dotted line for $R a / R a_{\mathrm{c}}=16.8$ gives the observation inferior boundary of the chaotic spectrum (Spectrum IV).]

Le temps de diffusion thermique de notre cavité étant de $\tau=17 \mathrm{~s}$, on a comme loi pour la fréquence adimensionnelle $\omega_{1}=f_{1} / \tau^{-1}$ :

$$
\omega_{1}=0,25 \frac{R a}{R a_{\mathrm{c}}}+3,6 .
$$

4. Conclusion. - Les résultats obtenus montrent clairement que la transition vers la turbulence pour l'air dans les cavités à facteur d'aspect élevé $\left(\Gamma_{x}=14\right)$, se fait avec une série de régimes dépendant du temps qualitativement comparables à ceux observés pour l'hélium liquide et l'huile silicone dans des cavités à faible facteur d'aspect $\left(\Gamma_{x}\right.$ de l'ordre de 1$)$. 
A l'examen de nos résultats expérimentaux, on ne peut invoquer le facteur d'aspect comme seul paramètre d'interprétation des observations de Ahlers et Behringer [4] faites en cavité cylindrique pour des facteurs d'aspect encadrant le nôtre. Il est naturel de penser que le nombre de degrés de liberté, lié à la géométrie du système, a son rôle à jouer.

\section{Bibliographie}

[1] Dubois, M. et Berge, P., J. Physique 42 (1980) 167-174.

Dubois, M. et Berge, P., Phys. Lett. 76A (1980) 53-56.

Berge, P. et Dubois, M., J. Physique Lett. 40 (1979) L-505-L-509.

[2] Maurer, J. and LibChaber, A., J. Physique Lett. 41 (1980) L-515-L-518.

Maurer, J. and Libchaber, A., J. Physique Lett. 40 (1979) L-419.

Maurer, J. and Libchaber, A., J. Physique Lett. 39 (1978) L-369.

[3] Pomeau, Y. and Manneville, P., Phys. Lett. 75A (1980) 296-298.

[4] Ahlers, G. and Behringer, R. P., Phys. Rev: Lett. 40 (1978) 712-715.

[5] Gollub, J. P. and Benson, S. V., J. Fluid Mech. 100 (1980) 449.

[6] Oertel Jr., H., Natural Convection in Enclosures. Presented at the 19th National Heat Transfer Conference Orlando, Florida. Edited by K. E. Torrance (Cornell University) 1980, p. 11-16.

[7] Willis, G. E. and Deardorff, J. W., J. Fluid Mech. 44 (1970) 661. 